\title{
Comment on: "The impact of COVID policies on acute ophthalmology services-experiences from Moorfields Eye Hospital NHS Foundation Trust"
}

\author{
Sarah Schimansky $\mathbb{1}^{1} \cdot$ Ushna Javed $^{2} \cdot$ Quresh Mohamed $^{2}$ \\ Received: 19 June 2020 / Revised: 15 July 2020 / Accepted: 12 August 2020 / Published online: 21 August 2020 \\ (c) The Royal College of Ophthalmologists 2020
}

\section{To the Editor:}

We have read with great interest the recent article by Wickham et al. on 'The impact of COVID policies on acute ophthalmology services-experiences from Moorfields Eye Hospital NHS Foundation Trust' [1]. During the pandemic, the authors noted a $>50 \%$ decline in attendances to their Accident \& Emergency (A\&E) departments and a sharp decline in new referrals for age-related macular degeneration (ARMD) with an almost 25\% 'Did Not Attend' (DNA) rate by patients already known to the ARMD service [1].

To gain further insight into the impact of COVID-19 on urgent wet ARMD services across the UK, we conducted a self-administered Qualtrics ${ }^{\mathrm{XM}}$ survey among retinal consultants. Individual survey links were sent to 53 consultants at 46 institutions. We received 18 responses (34\% response rate) from hospitals with rapid access wet ARMD services across England and Northern Ireland (Fig. 1). No responses were received from units in Wales or Scotland. Eight units (44.4\%) performed optical coherence tomography imaging on fellow eyes during the lockdown period (Fig. 2).

Prior to the Covid-19 pandemic the majority (66.7\%) of medical retina departments received more than 20 new urgent ARMD referrals per month, while in April and May 2020 most units $(83.3 \%)$ reported less than 20 monthly referrals (Fig. 2). In addition, an increase in the DNA or cancellation rate of existing ARMD patients was noted by $77.8 \%$ of units. Four respondents estimated this rate to be

Sarah Schimansky

sarah.schimansky@nhs.net

1 Department of Ophthalmology, The Royal United Hospitals Bath NHS Foundation Trust, Bath, UK

2 Department of Ophthalmology, Gloucestershire Hospitals NHS Foundation Trust, Cheltenham, UK equivalent to $20-30 \%$ of their booked appointments, while three consultants reported cancellations and DNAs in the region of more than 100 patients per month. One unit kept their cancellation rate at $\sim 10 \%$ by proactively ringing patients to explain their new protocols.

To facilitate the safe continuation of rapid access wet ARMD services, some units cancelled routine monitoring clinics, moved to peripheral sites, put in place physical distancing or one-stop virtual clinics. However, some respondents noted that the need for physical distancing has reduced their capacity, while others reported that some patients declined to attend due to anxiety, shielding or the lack of transport options.

Our results support findings by Wickham et al. [1] by showing a noticeable decline in new ARMD referrals in England and Northern Ireland during the COVID-19 pandemic. This may partially be explained by reduced access to and referrals by community optometrists and General Practitioners [2]. In addition, patients have been less likely to seek medical attention due to fear of contracting COVID19 or due to government advice on shielding. This change in health-seeking behaviour is reflected in a $56.6 \%$ year-onyear reduction in attendance at $\mathrm{A} \& \mathrm{E}$ attendance in England in April 2020 [3].

While our sample size does not allow for generalisation, it is important to recognise that the COVID-19 pandemic has had a marked impact on the provision of wet ARMD care and, subsequently, the visual morbidity of patients in the UK. Further studies are needed to quantify this impact and identify strategies to mitigate service disruptions during future pandemics.

\section{Compliance with ethical standards}

Conflict of interest The authors declare that they have no conflict of interest. 
Where is your unit located?

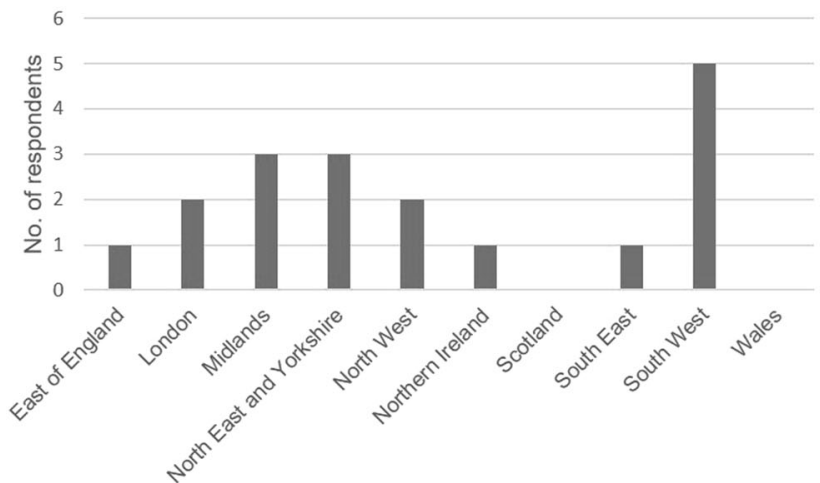

How large is your unit's catchment area?

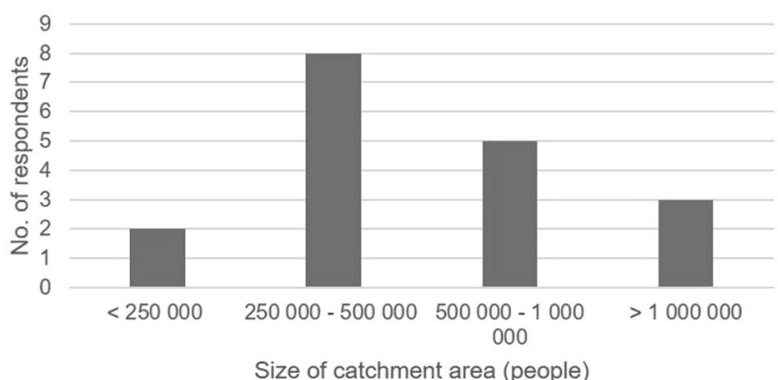

Fig. 1 Demographic information of the respondents' units. Distribution of answers to survey questions.

In the last month, has your unit performed OCTs on

fellow eyes of patients under treatment for wet ARMD?

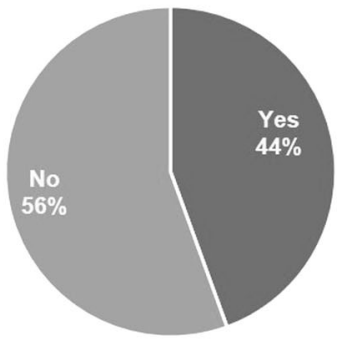

Prior to the Covid-19 lockdown, how many patients on average were referred to the service per month?

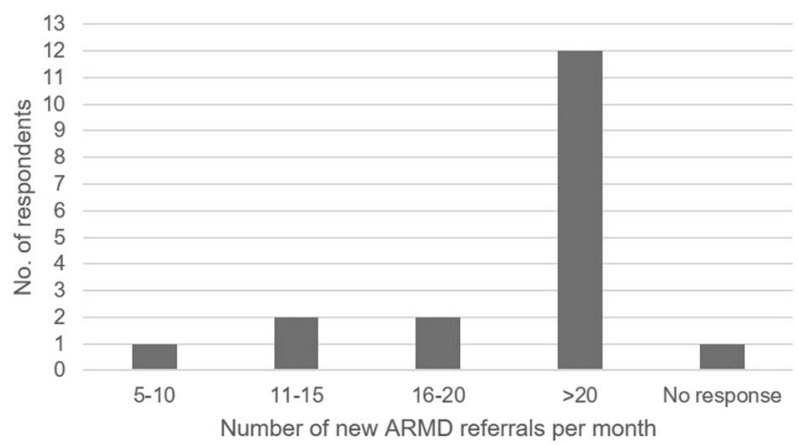

In the last month, how many patients have been referred to the service?

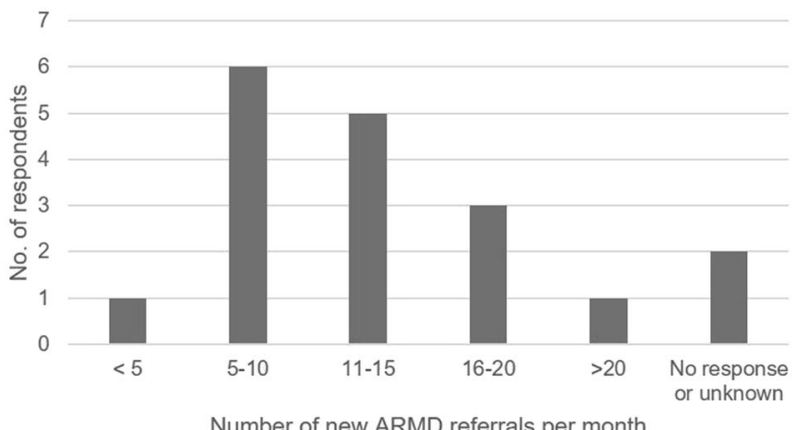

Number of new ARMD referrals per month

Fig. 2 Age-related macular degeneration services before and during the COVID-19 pandemic. Distribution of answers to survey questions.

Publisher's note Springer Nature remains neutral with regard to jurisdictional claims in published maps and institutional affiliations.

\section{References}

1. Wickham L, Hay G, Hamilton R, Wooding J, Tossounis H, da Cruz $\mathrm{L}$, et al. The impact of COVID policies on acute ophthalmology services-experiences from Moorfields Eye Hospital NHS Foundation Trust. Eye. 2020;34:1189-92.
2. Nuffield Trust. Chart of the week: The alarming drop in referrals from GPs to hospital services since the Covid-19 outbreak. 2020. https://www.nuffieldtrust.org.uk/resource/new-chart-of-the-weekthe-alarming-drop-in-referrals-from-gps-to-hospital-services-sincethe-covid-19-outbreak.

3. NHS England. A\&E Attendances and Emergency Admissions April 2020 Statistical Commentary. 2020. [cited 12 June 2020]. [https://www.england.nhs.uk/statistics/wp-content/uploads/sites/2/ 2020/05/Statistical-commentary-April-2020-jf8hj.pdf]. 